\title{
Transition Formation in Heat-Assisted Magnetic Recording
}

\author{
S. J. Greaves ${ }^{1, *}$, T. Mizuno ${ }^{2}$ and H. Muraoka ${ }^{1}$ \\ ${ }^{1}$ Tohoku University, Aoba-ku, Sendai 980-8577, Japan, ${ }^{2}$ TDK Corporation, Saku City, 385-8555, Japan \\ * Tel: +81-22-217-5458, Fax: +81-22-217-5496, E-mail: simon@ riec.tohoku.ac.jp
}

The effect of head field gradients and thermal gradients in heat assisted magnetic recording is investigated. When recording at a constant temperature, or in a constant external field, clear correlations between the recording performance and the head field gradient or thermal gradient can be found. Thermally assisted recording with a write head is much more complex and higher gradients do not necessarily translate into higher SNR.

Key words: perpendicular media, thermally assisted magnetic recording, simulation

\section{Introduction}

To realise high density magnetic recording it is necessary to reduce the size of the grains in the recording layer. Unfortunately, smaller grains are more susceptible to spontaneous, thermally induced reversals of their magnetisation. The energy barrier of a single phase, single domain grain is given by $\Delta E=K_{u} V$, where $K_{u}$ is the uniaxial anisotropy and $V$ the grain volume. If $K_{u}$ is increased thermal stability can be restored, but increasing $K_{u}$ also increases the anisotropy field, $H_{k}$, and will eventually make it impossible to switch the grain with the field from a write head. Thermally-assisted recording allows the use of magnetic materials with high uniaxial anisotropy: during recording the medium is locally heated, reducing $H_{k}$ and allowing the write head to record data bits ${ }^{1)}$.

If data bits are to be placed close together, sharp transitions between the bits are required. In conventional recording transition sharpness is influenced by the head field gradient in the downtrack (y) direction, $d H_{h} / d y^{2)}$. In thermally-assisted recording an additional gradient arises due to the temperature dependence of the anisotropy field, $d H_{k} / d T$ and the down-track variation of temperature, $d T / d y$. The total field gradient is then ${ }^{3)}$

$$
\frac{d H}{d y}=\frac{d H_{h}}{d y}+\frac{d H_{k}}{d T} \cdot \frac{d T}{d y}
$$

The second term in eq. 1 has the potential to be much larger than the head field gradient, suggesting that thermally-assisted recording may have an advantage over conventional recording. In this paper the effects of these gradients on the written transitions and recording performance are investigated using micromagnetic modelling.

\section{The Models}

A micro-magnetic model based on the LLG equation was used to determine the magnetisation of recording media. The media consisted of a $15 \mathrm{~nm}$ thick magnetic recording layer, a $27 \mathrm{~nm}$ interlayer and a $60 \mathrm{~nm}$ soft underlayer (SUL) on top of a glass substrate. The recording layer comprised single phase grains with an average pitch of $5.5 \mathrm{~nm}$, an average size of $4.6 \mathrm{~nm}$ and a size dispersion (standard deviation) of $9 \%$. The recording layer was divided into three $5 \mathrm{~nm}$ layers to allow incoherent magnetisation reversal. There was no inter-granular exchange coupling, but the vertical exchange coupling constant between the layers within each grain was $10 \mathrm{erg} / \mathrm{cm}^{2}$ at zero Kelvin and varied according to $\left(M_{s}(T) / M_{s}(0)\right)^{2}$. At a temperature of zero Kelvin the saturation magnetisation, $M_{s}(0)$, was $700 \mathrm{emu} / \mathrm{cm}^{3}$ and $K_{u}(0)$ was $15 \times 10^{6}$ $\mathrm{erg} / \mathrm{cm}^{3}$. The temperature variation of $K_{u}$ was defined by

$$
K_{u}(T)=K_{u}(0)\left(\frac{\cosh (\chi)-\cosh \left(\chi T / T_{c}\right)}{\cosh (\chi)-1}\right)
$$

where $T$ is the temperature, $T_{c}$ is the Curie temperature $(600 \mathrm{~K}$ in this paper) and $\chi$ is a parameter which controls the slope of $d K_{u}(T) / d T$ near to the Curie temperature. The function is similar to a Langevin function and is used for convenience since the expression to the right of $K_{u}(0)$ in eq. 2 varies between 1 and zero as $T$ varies between zero and $T_{c}$.

Although there were no dispersions of $K_{u}$ or easy axes among the grains in each medium, a significant switching field distribution was induced by the grain size distribution and thermal effects. For a fixed grain size a switching field distribution arises due to the effect of temperature. The grain size distribution further broadens the switching field distribution as smaller grains tend to reverse in lower fields than larger grains.

Heating and thermal conduction were modelled using the heat flow equation. Thermal conductivities, $K$, and specific heat capacities, $C$, of the various layers are listed in table 1 . Heating was provided by a laser heat source with a Gaussian intensity distribution and a spot diameter (half-width) of $60 \mathrm{~nm}$. The laser wavelength was $413 \mathrm{~nm}$ and the absorption coefficient was 2.1 for the recording layer, interlayer and soft underlayer and zero for the glass substrate. The laser power was selected such that the maximum temperature in the centre of the recording layer was $645 \mathrm{~K}$. The head-medium velocity was $23.5 \mathrm{~m} / \mathrm{s}$ and the head field rise time was 0.12 ns (zero to $90 \%$ of peak output).

\section{Head field gradient}

The effect of the head field gradient on the recording performance was investigated by maintaining a constant temperature 
Table 1 Thermal conductivities and specific heat capacities used in the modelling

\begin{tabular}{lcc}
\hline Layer & $K(\mathrm{~W} / \mathrm{cmK})$ & $C\left(\mathrm{~J} / \mathrm{cm}^{3} \mathrm{~K}\right)$ \\
\hline RL & 0.924 & 3.417 \\
IL & 1.170 & 2.850 \\
SUL & 1.000 & 2.500 \\
Glass & 0.015 & 2.000 \\
\hline
\end{tabular}

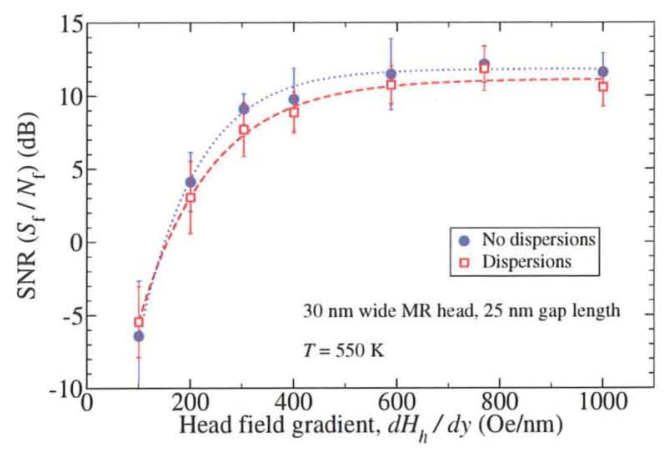

Fig. 1 SNR of $1270 \mathrm{kfci}$ tracks written with various head field gradients.

and using an artificial head field distribution (probe head) with the following properties: $H_{x}=0, H_{y}=0$ and $H_{z}=10 \mathrm{kOe}$ over a region $40 \mathrm{~nm}$ wide and $100 \mathrm{~nm}$ long. Here, $z$ is the axis perpendicular to the plane of the medium. At the boundary of the 40 $\mathrm{nm} \times 100 \mathrm{~nm}$ region the head field decayed to zero with a constant gradient which was varied from $100 \mathrm{Oe} / \mathrm{nm}$ to $2000 \mathrm{Oe} / \mathrm{nm}$. Tracks were written at $1270 \mathrm{kfci}$ (20 $\mathrm{nm}$ bit length) and the SNR was calculated from readback waveforms obtained using reciprocity ${ }^{4)}$. The computed sensitivity function of a $30 \mathrm{~nm}$ wide magneto-resistive (MR) read head with a shield-shield spacing of $25 \mathrm{~nm}$ was used for the reciprocity calculation. The temperature was fixed at $550 \mathrm{~K}$, making the medium $H_{k}$ low enough for the probe head to be able to write on the medium, and tracks were written using a range of head field gradients. The results are shown in fig. 1 where each point represents the average of five simulations and the error bars represent one standard deviation of the SNR values. From $100 \mathrm{Oe} / \mathrm{nm}$ up to $600 \mathrm{Oe} / \mathrm{nm}$ the SNR increased with increases in the head field gradient; above 600 $\mathrm{Oe} / \mathrm{nm}$ the SNR was almost constant. Introducing a $10 \%$ dispersion of $K_{u}$ and a $3^{\circ}$ dispersion of easy axes slightly reduced the SNR. At $550 \mathrm{~K}$ thermal instability was a problem and many grains reversed at random, even in areas where no head field was applied and this reduced the SNR of the written tracks. In media with high SNR, transition noise was dominant. For lower values of SNR, e.g. below $10 \mathrm{~dB}$ at $1270 \mathrm{kfci}$, noise from reversed grains within written bits was also significant.

\section{Thermal gradients}

The effect of thermal gradients was investigated using a uniform magnetic field with a maximum magnitude of $10 \mathrm{kOe}$ and

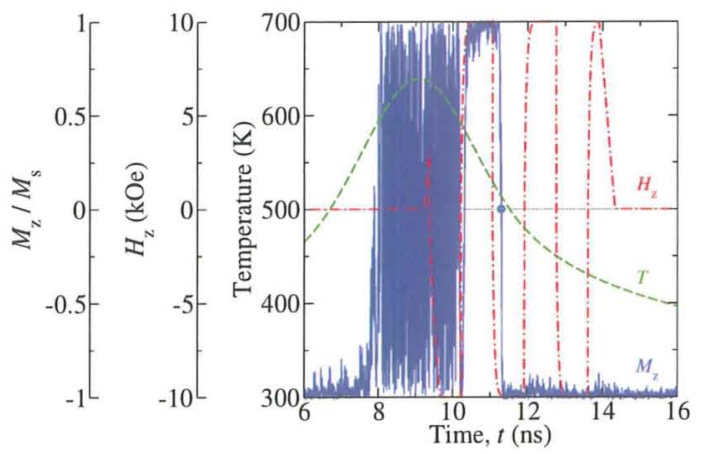

Fig. 2 Grain magnetisation (normalised $z$ component), temperature and head field during a simulation.

a rise time of $0.12 \mathrm{~ns}$. Since the magnetic field was the same at every point, transitions were formed by the thermal gradients alone. The two thermal gradients $d H_{k} / d T$ and $d T / d y$ were varied independently. $d H_{k} / d T$ was changed via the parameter $\chi$ in eq. 2 and $d T / d y$ was controlled by adjusting the thermal conductivity of the SUL from $0.5 \mathrm{~W} / \mathrm{cmK}$ to $3 \mathrm{~W} / \mathrm{cmK}$. The laser power was adjusted in each case such that the maximum temperature in the centre of the recording layer was $645 \mathrm{~K}$.

The data were analysed by examining the magnetisation of a set of grains in the centre of the recording layer. An example of the behaviour of a random grain is shown in fig. 2. In this example the external field was supplied by a single pole head, which will be discussed in the following section, but the method of analysis was the same. As the laser moved along the medium the temperature of the grain increased, peaked and decreased. When the temperature of the grain exceeded the Curie temperature $(600 \mathrm{~K})$ the magnetisation of the grain became unstable. Note that the $z$ component of the magnetisation vector shown in fig. 2 is normalised to unity. Once the medium cooled below $T_{c}$ the grain magnetisation was influenced by the head field, since $H_{k}$ was small. The thermal gradients were evaluated the point where the grain reversed for the final time, just after $t=11 \mathrm{~ns}$ and indicated by the point at $M_{z} / M_{s}=0$ in fig. 2 . Given the grain temperature $T, d H_{k} / d T$ was calculated from eq. 2 and $d T / d y$ was obtained from the down-track temperature distribution at that point, leading to $d H_{k} / d y$.

Tracks were written at $1270 \mathrm{kfci}$ and the SNR calculated with the MR read head. Fig. 3 shows the results for media with different values of SUL thermal conductivity. Each point on a curve with the same thermal conductivity represents a different value of $\chi$ in eq. 2. For each written track about 80 grains were monitored and five tracks were written for each point shown in fig. 3 . The standard deviations of $d H_{k} / d y$ were typically around $300 \mathrm{Oe}$ and error bars are omitted from fig. 3 for clarity. When $d H_{k} / d y$ was below $1000 \mathrm{Oe} / \mathrm{nm}$ there was a linear relationship between SNR and $d H_{k} / d y$ i.e. a minimum value of $d H_{k} / d y$ was needed to obtain a particular value of SNR. In this region similar SNR values were obtained regardless of whether $d H_{k} / d T, d T / d y$ or both 


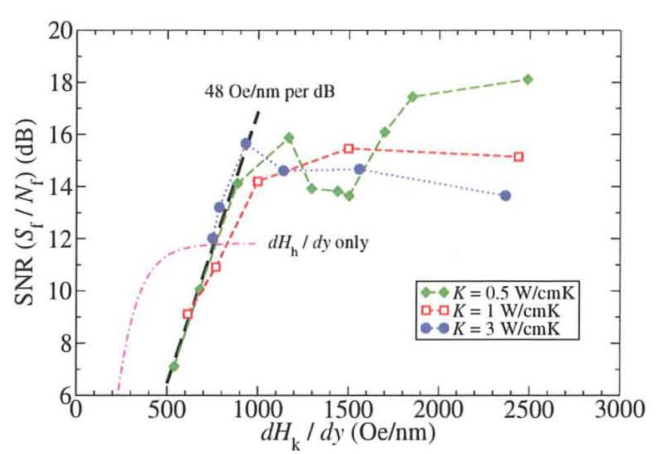

Fig. 3 SNR of 1270 kfci tracks vs. $d H_{k} / d y$. Uniform head field.

terms were varied. For larger values of $d H_{k} / d y$ different trends of SNR were observed, depending on the thermal conductivity of the SUL. Once $d H_{k} / d y$ exceeded $2000 \mathrm{Oe} / \mathrm{nm}$ media with lower values of $d T / d y$ in the recording layer had higher SNR.

Fig. 3 also shows a curve representing the "No dispersions" data from fig. 1 , indicated as " $d H_{h} / d y$ only". For head field gradients up to $700 \mathrm{Oe} / \mathrm{nm}$ higher SNR could be obtained than when thermal gradients of the same magnitude were used. Ultimately, thermal recording permitted higher values of SNR as thermal instability was not a problem once grains had cooled after writing.

\section{Recording with a single pole head}

For recording with both head and thermal gradients the magnetic field distribution from a single pole type (SPT) head was used. The head was modelled as a uniformly magnetised cuboid $40 \mathrm{~nm}$ wide, $100 \mathrm{~nm}$ long, $500 \mathrm{~nm}$ high and with a saturation magnetisation of $1910 \mathrm{emu} / \mathrm{cm}^{3}$. The spacing between the head and the medium was $5 \mathrm{~nm}$, giving a maximum field in the centre of the recording layer of $7.3 \mathrm{kOe}$. The maximum effective head field ${ }^{5)}$ in the centre of the recording layer was about $10 \mathrm{kOe}$; this was calculated by considering the angular variation of the switching field of a single grain at $574 \mathrm{~K}$. The temperature of $574 \mathrm{~K}$ was chosen because this was the average temperature at which grains switched for the final time during the recording simulations.

When recording with a SPT head field, the relative positions of the head and heat spot are important. Fig. 4 shows the SNR of tracks written by a single pole head at two linear densities for various down-track offsets between the centre of the heat spot and the centre of the SPT head. The extent of the main pole is indicated by the shaded area in fig. 4 and the points represent the centre of the heat spot. Points within the shaded area are physically impossible to manufacture as the heat source must be co-located with the head. Fig. 4 shows that good SNR can be maintained if the centre of the heat spot is located no more than $31 \mathrm{~nm}$ in front of the leading edge of the main pole. If the heat spot is further from the main pole the medium is too cool, and the anisotropy field too high, for the head to be able to write bits when the medium enters the head field. Locating the heat spot behind the main pole is not a possible solution as the head field is

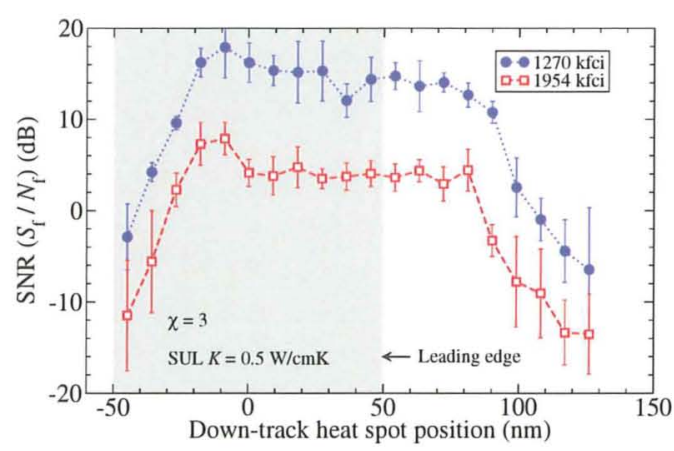

Fig. 4 SNR vs. down-track position of heat spot, measured from the centre of the SPT head. The shaded area indicates the position of the SPT head.

too small to influence the magnetisation once the medium cools below the Curie temperature.

Fixing the position of the heat spot $81 \mathrm{~nm}$ in front of the centre of the main pole ( $31 \mathrm{~nm}$ from the leading edge), tracks were written in media with various values of $d H_{k} / d y$. In the calculations $d H_{k} / d y$ was measured when the grains switched for the final time and was controlled through the $\chi$ parameter. Fig. 5 shows the SNR of the written tracks versus the total gradient $d H / d y$. The total gradient includes the head field gradient, $d H_{h} / d y$, which is plotted as a separate curve in fig. 5. The average grain $H_{k}$ value during switching was closely related to the magnitude of the effective head field and not to the magnitude of the vertical component of head field, suggesting that the effective head field determined grain switching. Therefore, $d H_{h} / d y$ in fig. 5 is the gradient of the effective head field, calculated using the angular variation of switching fields at $574 \mathrm{~K}$. The head field gradients were all negative because they were measured near the leading edge of the main pole. The variation in head field gradient reflects a change in the position of the written transitions as $d H_{k} / d y$ was varied. The SNR was maximised when $d H / d y$ was around $1000 \mathrm{Oe} / \mathrm{nm}$ : higher gradients decreased the SNR, possibly because extremely large values of $d H_{k} / d y$ do not allow sufficient time for grains to switch in the head field before the grain $H_{k}$ becomes much larger than the head field. These results suggest that the best recording performance will not necessarily be obtained by maximising $d H / d y$, but that other factors should also be taken into consideration. This result differs from those in sections 3 and 4, where the SNR tended to saturate as the head field gradient or thermal gradient was increased. One reason for the difference is that as $\chi$ and $d H_{k} / d y$ increase, the position of the transitions moves closer to the centre of the heat spot. When using a SPT head with a heat spot located in front of the leading edge of the main pole this means that the head field decreases as $d H_{k} / d y$ increases, resulting in lower SNR. This effect is not apparent in fig. 3 as the external magnetic field is uniform over the entire medium.

Correlations between $d H / d y$ and the SNR were investigated for various recording conditions (heat spot position, linear den- 


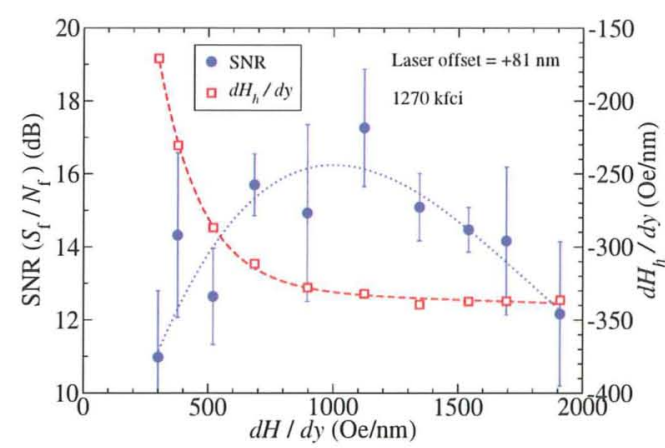

Fig. 5 SNR vs. total gradient $d H / d y$ for a heat spot $31 \mathrm{~nm}$ in front of the leading edge of the main pole. $d H / d y$ was controlled by changing $\chi$.

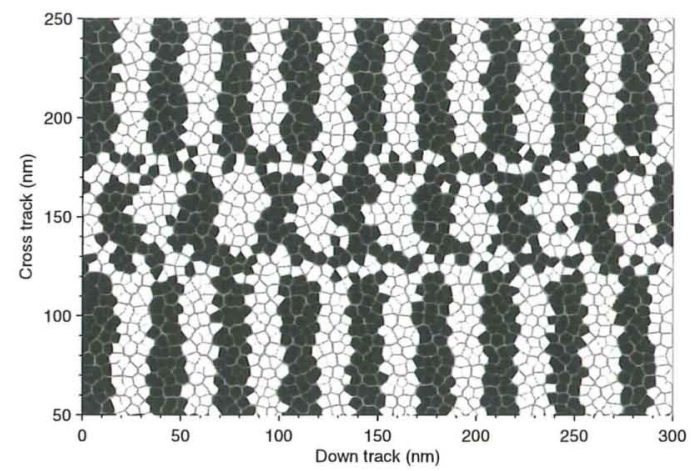

Fig. 6 Written track for $d H / d y=1120 \mathrm{Oe} / \mathrm{nm}$. Heat spot is 31 $\mathrm{nm}$ in front of the leading edge of the main pole.

sity, $\chi$, head width), but no clear relationship between the total gradient $d H / d y$ and the SNR of the written tracks could be established. It is possible that any such relationship was masked by variations in the magnitude of the head field at the writing point as the heat spot - head centre offset was altered.

A written track corresponding to the conditions which maximised the SNR in fig. 5 is shown in fig. 6. Transition curvature induced by the temperature distribution was partially mitigated by the curvature of the head field which, at the leading edge, is opposite to the contours of equal temperature. The written track width was about $50 \mathrm{~nm}$ under these conditions which, at a linear density of $1954 \mathrm{kfci}$, equates to an areal density of $1 \mathrm{~Tb} / \mathrm{in}^{2}$.

The width of the write head was increased from $40 \mathrm{~nm}$ to 100 $\mathrm{nm}$ in $20 \mathrm{~nm}$ steps to examine the effect on the recording performance. The maximum effective head field in the centre of the recording layer increased from $10 \mathrm{kOe}$ with the $40 \mathrm{~nm}$ wide head to $13 \mathrm{kOe}$ with the $100 \mathrm{~nm}$ wide head, with a consequent increase in the head field gradient. Fig. 7 shows the SNR of the resulting tracks. For all of the head widths studied locating the heat spot $31 \mathrm{~nm}$ in front of the leading edge of the main pole gave adequate recording performance and the maximum SNR was obtained when the heat spot was $9 \mathrm{~nm}$ behind the centre of the main pole. Significant differences in recording performance were observed when the heat spot was $36 \mathrm{~nm}$ in front of the centre of the

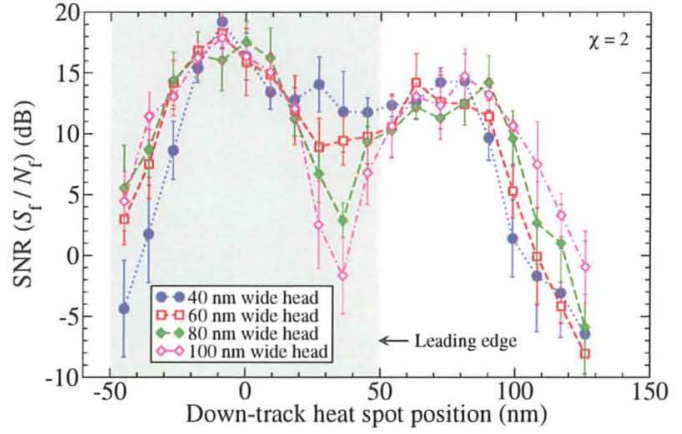

Fig. 7 Effect of write head width on SNR for various down-track heat spot positions. $1270 \mathrm{kfci}$ tracks.

main pole. Comparing the data for the $40 \mathrm{~nm}$ and $100 \mathrm{~nm}$ wide heads at this point, the average values of $d H / d y$ and $d H_{k} / d y$ were found to be higher for the narrower head. The average head field gradient at this point was close to zero for both heads, but the distribution of $d H_{h} / d y$ was significantly broader for the $100 \mathrm{~nm}$ wide head. Wider write heads can be beneficial if the heat spot is in front of the leading edge of the main pole: for offsets between $99 \mathrm{~nm}$ and $126 \mathrm{~nm}$ the SNR increased as the width of the write pole increased. Part of this increase may be due to wider written tracks and may not translate into higher areal densities.

\section{Conclusion}

For recording with head field gradients only, or thermal gradients only it was possible to find clear correlations between the quality of recorded tracks and the head field gradient. For thermally assisted recording with a single pole head the relationship between the total gradient $d H / d y$ and SNR is much more complex and influenced by numerous factors other than the head and thermal gradients. With the correct choice of parameters it is possible to obtain good recording performance at high areal densities with a physically feasible configuration.

\section{References}

1) M. H. Kryder, E. C. Gage, T. W. McDaniel, W. A. Challener, R. E. Rottmayer, G. Ju, Y. T. Hsia, and M. F. Erden, "Heat assisted magnetic recording," Proc. IEEE, vol. 96, pp. 18101835, Nov. 2008.

2) K. Miura, H. Muraoka, and Y. Nakamura, "Effect of head field gradient on transition jitter in perpendicular magnetic recording," IEEE Trans. Magn., vol. 37, pp. 1926-1928, July 2001.

3) J. U. Thiele, K. R. Coffey, M. F. Toney, J. A. Hedstrom, and A. J. Kellock, "Temperature dependent magnetic properties of highly chemically ordered $\mathrm{Fe}_{55-x} \mathrm{Ni}_{x} \mathrm{Pt}_{45} \mathrm{~L} 1_{0}$ films," J. Appl. Phys, vol. 91, pp. 6595-6600, May 2002.

4) J. C. Mallinson, Magneto-resistive heads: Fundamentals and applications. Academic Press, New York, 1996.

5) A. Shukh, "Writability of perpendicular recording heads," IEEE Trans. Magn., vol. 40, pp. 2585-2587, July 2004. 